\title{
Various Methods for Measurement of Resistive Leakage Current of Metal Oxide Surge Arrester
}

\author{
Prof. Samruddhi Shaha, Yaseen Khan Anwar Khan Dakhani
}

Department of Electrical Engineering, N.K. Orchid College of Engineering and Technology, Solapur, Maharashtra, India

\section{ABSTRACT}

\section{Article Info}

Volume 8, Issue 4

Page Number: 81-89

\section{Publication Issue :}

July-August-2021

\section{Article History}

Accepted : 06 July 2021

Published: 13 July2021
Metal Oxide Surge Arrester (MOSA) does not require any ordinary maintenance. But MOSA is get affected from ageing due to the effect of discharging impulse currents, uneven heating and internal partial discharges. A leading cause of deterioration is ingress of moisture. The degradation of MOSA also correlated with the ageing level of the arrester. This is called degradation under surge. The ageing level with the degradation of MO surge arrester or the leakage current of the surge arrester is related to each other. The leakage current is directly proportional to the ageing level. If the ageing level is raise, the leakage current also will increase. So, various methods for determination of the condition of $\mathrm{ZnO}$ surge arrester by means of measurement of the arrester leakage current are discussed. A MSCM method, which is based on leakage current of the surge arrester presented. The measuring instrument Leakage Current Monitor is also discussed.

Keywords : Metal Oxide Surge Arrester, Leakage Current, Resistive Leakage Current, Various Technique Of Measurement Of Resistive Leakage Current.

\section{NOMENCLATURE:}

\begin{tabular}{clcl}
\hline $\mathbf{I}_{\mathbf{t}}$ & Total Leakage Current $(\mathrm{mA})$ & $\boldsymbol{\theta}_{\mathbf{k}}$ & k order harmonics voltage phase angle \\
\hline $\mathbf{I}_{\mathbf{r}}$ & Resistive Leakage Current $(\mu \mathrm{A} / \mathrm{mA})$ & $\mathrm{f}_{\mathrm{k}}$ & Harmonic component of capacitive leakage current \\
$\mathbf{I}_{\mathbf{c}}$ & Capacitive Leakage Current $(\mathrm{mA})$ & $\mathrm{I}_{\mathrm{r} 3}$ & Third harmonics of the resistive current \\
$\mathbf{C}$ & Material constant & $\mathrm{I}_{\mathrm{t} 3}$ & Third harmonics of total leakage current \\
$\boldsymbol{\alpha}$ & Temp \& Voltage dependent constant & $\mathrm{k}$ & Compensation Ratio \\
$\boldsymbol{G}$ & Amplifier gain & $K_{3}, K_{1}$ & Constant Ratio \\
$\boldsymbol{V}$ & Applied voltage $(\mathrm{kV})$ & $I_{p 1}$ & Fundamental probe current \\
$\boldsymbol{\varphi}_{\mathbf{1}}$ & Power angle & $I_{p 3}$ & Third harmonic probe current \\
$\boldsymbol{U}_{\boldsymbol{k}}$ & k order harmonics voltage & $I_{n}$ & Neutral Current \\
\hline
\end{tabular}

Copyright: () the author(s), publisher and licensee Technoscience Academy. This is an open-access article distributed under the terms of the Creative Commons Attribution Non-Commercial License, which permits unrestricted non-commercial use, distribution, and reproduction in any medium, provided the original work is properly cited 


\section{INTRODUCTION}

Zinc oxide surge arresters behave as an insulator at normal line to earth voltage. They have very high impedance resulting in leakage current with few $\mathrm{mA}$. During over voltage events the metal oxide surge arrester limits the voltage to an almost constant value, even if the discharge current increases extremely. This capability has been found to remain unchanged during its full life, but the insulation performance at operation voltage may subject to change.

Metal oxide surge arrester requires no ordinary maintenance. Several ways of monitoring of metal oxide surge arrester are there, most of methods are depends on the leakage current [1]. There are several methods of separating the resistive leakage current from the total leakage current of $\mathrm{ZnO}$ surge arresterThe total leakage current of a $\mathrm{MO}$-surge arrester is composed of a capacitive and a resistive part, where the capacitive component is much bigger than the resistive. MO-surge arresters are known to exhibit an increase in resistive leakage current in relation with the arrester's operating time. Furthermore, the increase rate of resistive leakage current increases, with the increase of the applied voltage and the increase of ambient temperature. Any change, degradation or aging in the arrester results in an increase to the resistive component of the leakage current, while the capacitive part has little change. An increase of the resistive current can be considered as an indicator of the arresters condition, and with the continued operation time it can cause failures or permanent degradation. The leakage current is directly proportional to the ageing level. If the ageing level is raise, the leakage current also increases [4]. The third harmonic of the resistive leakage current is responsible to the degradation of $\mathrm{ZnO}$ surge arrester. So the resistive component of leakage current is a good indicator of surge arrester condition. In this paper different method of measurement of resistive leakage current is discussed. The Modified Shifted
Current Method is discussed to separate resistive leakage current from total leakage current. The applied voltage is not necessary in this method to determine the resistive leakage current. There is no need to disconnect the surge arrester from main system while measuring process takes place.

\section{ELECTRICAL REPRESENTATION OF METAL OXIDE SURGE ARRESTER}

It is necessary to determine the V-I characteristic of surge arrester. In this the resistive component is predominant. The continuously operating voltage is in range of $0.7-0.8$ p. u. of rated voltage. Figure 1 shows the electrical representation of metal oxide surge arrester. [1]

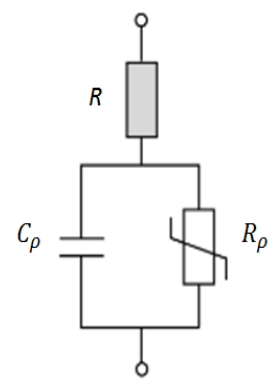

Figure 1 Simplified Equivalent Circuit of MOSA (Zno)

The parallel combination of capacitor $C_{\rho}$ and nonlinear resistor $R_{\rho}$, represents the barriers and the small resistance $R$, of the $\mathrm{ZnO}$ grains is in series with the parallel combination. Usually, $R$, is ignored except for very high arrester currents. The arrester current is separated into two parts, resistive $i_{r}$ and capacitive $i_{c}$ components. Normally the capacitance is $75 \rho \mathrm{F} / \mathrm{kV}^{2}$. Typically the value of leakage current is in $\mathrm{mA}$ depending on the rating of the surge arrester. The value of capacitive current is more which did not affect on the surge arrester failure. The resistive component of leakage current is in range of $\mu \mathrm{A}$ which affects strongly on the life of surge arrester.

$\mathrm{ZnO}$ material has high non-linear V-I characteristics and high energy absorption capability as their polycrystalline nature and the large number of 
intergranular barriers. The resistive characteristics of metal oxide surge arrester is expressed as,

$$
I_{r}=C V^{\alpha}
$$

In normal operating condition the value of $\alpha$ should be between 20 to 30 . And the resistive current should not increase above $3 \mathrm{~mA}$. It is critical condition for surge arrester when its resistive leakage current increases.

\section{VARIOUS METHODS FOR MEASUREMENT OF SURGE ARRESTER RESISTIVE LEAKAGE CURRENT}

The different methods are used to measure the resistive leakage current in normal operating conditions. The measuring techniques are divided basically in to two parts according to requirement of the input parameters such as applied voltage, leakage current.

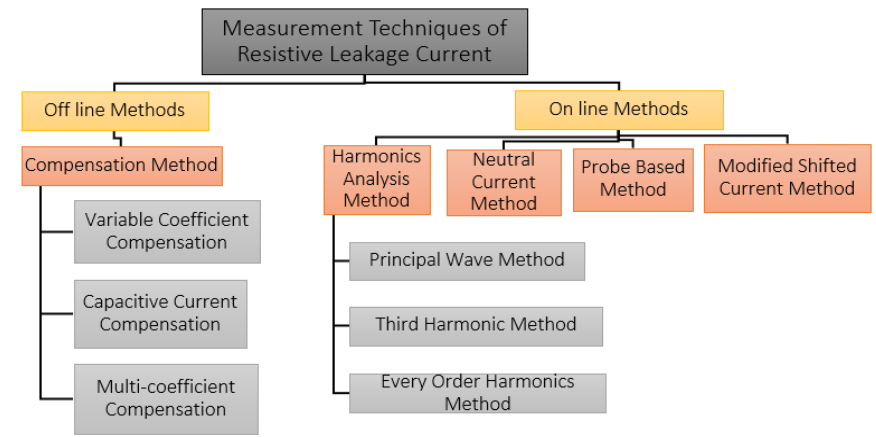

Figure 2 Various Methods to Measure Resistive Leakage Current

\subsection{Offline Measurement Techniques:}

In offline measurements, arrester is disconnected from the system and energized with a separate voltage source on site or in a laboratory. Offline measurements can be performed with voltage sources that are especially suited for this purpose, for example, DC test generators. Good accuracy may be obtained by using the offline methods, provided that a sufficiently high test voltage is used. The main disadvantages of this method are the cost of the required equipment and the need for disconnecting the arrester from the system.

\subsubsection{Compensation method [1][2]}

One of the most common and straight forward techniques for extracting the resistive leakage current (LC) for the purpose of condition monitoring is the compensation technique. Compensation method is the conventional monitoring methods for MOV surge arrester, the leakage current measuring apparatus of LCD-4 type made in japan which monitors the total leakage Current, the resistive current and power loss. In order to extract the resistive component from the total leakage current, the voltage across the arrester terminals is usually measured and used as a reference so that, based on the phase difference, the capacitive current component can be established. The resistive component is then obtained by just subtracting the capacitive component from the total leakage current.

Compensation method is the conventional monitoring method for separating the resistive leakage current of $\mathrm{ZnO}$ surge arrester. Measuring principle is based on the orthogonally between the resistive current and the capacitive leakage current. It satisfies the following equation,

$$
i_{r}=i_{t}-i_{c}
$$

If the applied voltage is a pure sine wave, the capacitive current component can be written as,

$$
i_{c}=G v
$$

The resistive current is in phase with the voltage and the capacitive component is orthogonal to the voltage. Therefore, these currents satisfy,

$$
\int_{0}^{2 \pi} \boldsymbol{i}_{c} \boldsymbol{i}_{r} d \omega t=0
$$

Combining equation (1), (2), (3)

$$
\int_{0}^{2 \pi} v_{s h}\left(i_{t}-G v_{s h}\right) d \omega t=0
$$

So the capacitive component of the leakage current is set completely to get the resistive component. Actual measurement of applied voltage is necessary so it is not suitable for online application. Measurement of total leakage current in substations or other installations is easily done using current shunts or 
current transformers, but the measurement of applied voltage to obtain the resistive LC make these techniques more appropriate in the laboratory rather than onsite due to the difficulty of measuring the voltage.

\subsubsection{Variable coefficient compensation [3]}

This method imports the harmonics analysis to reform compensation method, which adopts the variable compensating coefficient to balance the capacitive portion of every order harmonics current. Using phase vector diagram, the resistive current is

$$
\boldsymbol{i}_{r}=\boldsymbol{i}_{t}-\frac{\boldsymbol{i}_{t} \sin \varphi_{1}}{\boldsymbol{v}_{s h}} \sum_{k=1}^{n} k \times \boldsymbol{U}_{k} \cos \left(k \omega t+\boldsymbol{\theta}_{k}\right)
$$

where $I_{t 1}, \varphi_{1}, U_{s h}$ are amplitude of the principal wave current of the arrester, the power angle (angle difference between the principal wave voltage and the corresponding current), and the amplitude of the principal wave voltage.

\subsubsection{Multi-coefficient compensation [1]}

As regards the influence of the high order harmonic voltages, the multi-coefficient compensating factor $f_{k}$ is used to offset every order harmonic component of capacitive leakage current in this method. Then the resistive current is extracted.

$$
\begin{gathered}
i_{R}=i_{X}-\sum_{k=1}^{n} f_{k} \cos \left(k \omega t+\theta_{k}\right) \\
f_{k}=\frac{\int_{0}^{2 \pi} i_{X} * \cos \left(k \omega t+\theta_{k}\right) * d \omega t}{\int_{0}^{2 \pi} \cos \left(k \omega t+\theta_{k}\right) * d \omega t}
\end{gathered}
$$

The capacitive current is removed completely from the total leakage current the remaining is the resistive leakage current.

\subsection{Online Measurement Techniques:}

In online measurements, arrester is connected to the system and energized with the service voltage during normal operation. Measurements carried out online under normal service voltage are the most common method. In order to allow measurements of the leakage current that flows in the earth connection, the arrester must be equipped with an insulated earth terminal. Online leakage current measurements are usually made on a temporary basis using portable or permanently installed instruments. Portable instruments are usually connected to the earth terminal of the arrester by means of a clip-on, or permanently installed, current transformer.

\subsubsection{Harmonics analysis method}

The third harmonic analysis, which does not need reference voltage signal, is based on the fact that the leakage current contains harmonics, due to the nonlinearity of the voltage-current characteristic [4]. Due to the nonlinear resistance of MOV, the leakage current contains harmonics when the arrester is energized with a sinusoidal voltage since the amplitudes of the harmonic currents increase with the resistive component of the leakage current, the harmonic content can be used as an indicator of the arrester condition. Principal wave method, the third harmonic method and every order harmonics method are together called as harmonics analysis method. The schematic diagram on monitoring MOV surge arrester is seen as Figure 3

Main disadvantage of the method is the fact that the measurement is influenced by the voltage harmonics and the practically unknown temperature of the arrester. However, resistive current detection diagnostic methods have been improved and many researchers have presented advanced methods and convenient instruments.

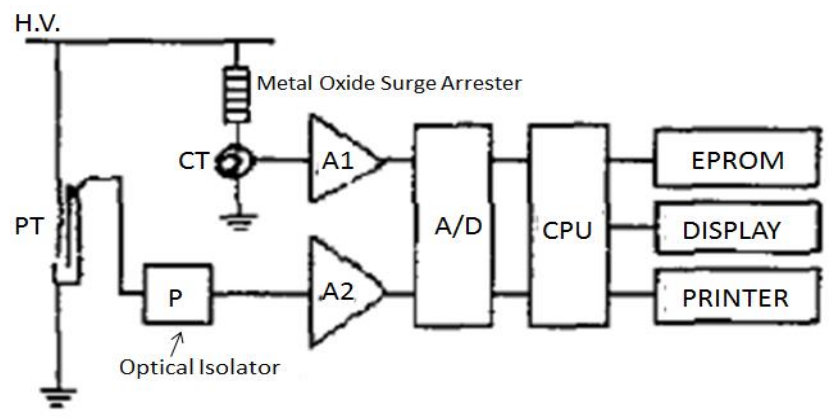

Figure 3 The Schematic Diagram of Harmonics Analysis Method 


\section{Principal wave method}

The pure sinusoidal voltage is applied across the arrester, the passing resistive current only contains the principal wave, and its value keeps fixed. In this way, the resistive proportion and its variation in the total leakage current can evaluate the working condition of MOV surge arrester.

\section{Third harmonic method}

The leakage current through MOV surge arrester has the third harmonic of resistive current. That is to say the third harmonic of the resistive current varies along with the third harmonic of the leakage current. According to the relation between the third harmonic and other order harmonics in resistive current, the resistive leakage current change can be observed. The harmonics in system voltage isn't counted in the two above methods, which will produce errors to the monitoring results.

\section{Every order harmonics method}

The presence of harmonics in the system voltage may with the harmonics generated by the nonlinear resistance of MOV surge arrester. The amount of system voltage harmonics varies with the type of load and the system voltage level. However, the resistive current characteristic of the arrester isn't affected by voltages harmonics in this method. Proceeded with FFT method, every order harmonics are obtained from the voltage and the total current. And the phase angle difference between the same order harmonic of resistive current and capacitive current is recognized as $90^{\circ}$. Then every order harmonic components of resistive current and capacitive current are also determined by the harmonics analysis.

\subsubsection{Probe current method}

This is a compensation technique originally proposed by Scandinavian. It uses the $3^{\text {rd }}$ harmonic component of the resistive leakage current as the indicator. This method is not sensitive to system harmonics, and the test result will not be influenced by the position of the probe.

This method for harmonics analysis of the leakage current is based on a probe current. During the measurement, a field probe is set on the arrester which produces the current. A relation between the resistive current and its third harmonic component is calculated [4].

The diagram of this method is shown in Fig. 4 from figure the total leakage current can be obtained with a shunt or a clamp type CT. The probe current $I_{p}$, can be obtained by introducing a capacitive probe. The capacitive is calculated as,

$$
\bar{I}_{r 3}=\bar{I}_{t 3}-\bar{I}_{c 3}
$$

Because of the difficulties in obtaining the capacitive current component, the probe current is used to derive $I_{c 3}$ so by putting

$$
K_{1}=\frac{I_{t 1}}{I_{p 1}}
$$

And

$$
K_{3}=\frac{I_{t 3}}{I_{p 3}}
$$

$I_{t 1}, I_{t 3}$ are fundamental and $3^{\text {rd }}$ harmonic leakage currents, and $I_{p 1}$ and $I_{p 3}$ are the fundamental and $3^{\text {rd }}$ harmonic probe currents.

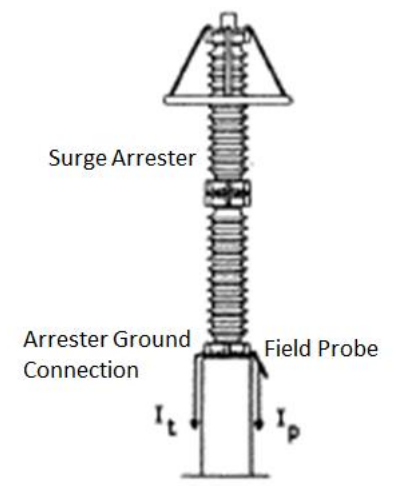

Figure 4: Probe Current Method

$$
I_{r 3}=I_{t 3}-k * \frac{I_{t 1}}{I_{p 1}} * I_{p 3}
$$

$I_{r 3}$ is the third harmonics of the resistive current leakage current. $I_{t}$ is principle wave current, $k$ is compensation coefficient which is constant and its value is between 0.7 to 1.0 
The probe picks up the $3^{\text {rd }}$ harmonic voltage component, which is then used to compensate for the influence of the $3^{\text {rd }}$ harmonic voltage. The $3^{\text {rd }}$ harmonic resistive leakage current component will not only be influenced by the fundamental and $3^{\text {rd }}$ harmonic voltages, but also by higher order harmonic voltages such as $5^{\text {th }}, 7^{\text {th }}$, and so on. In practice, the magnitude of these higher order harmonic voltages may be larger than that of the third order harmonic content. The influence of higher order harmonic voltages cannot be neglected. Another error is related to constant ratio of the two factors, $\mathrm{kl}$ and $\mathrm{k} 3$. However, this error can be eliminated by restoring the real data of electric field analysis for every configuration of MOSA.

\subsubsection{Neutral Current Method:}

As shown in Figure 5 the zero sequence leakage current of MOSA, $I_{n}$ can be obtained from the neutral line using a shunt or a suitable clamp type Current Transformer. If the MOSA in three phases are identical and three phase voltage is pure sinusoidal voltage and balanced, the sum of the positive and negative components of the leakage current will be zero, and the neutral current $I_{n}$ equals to $3 I_{t 3}$.

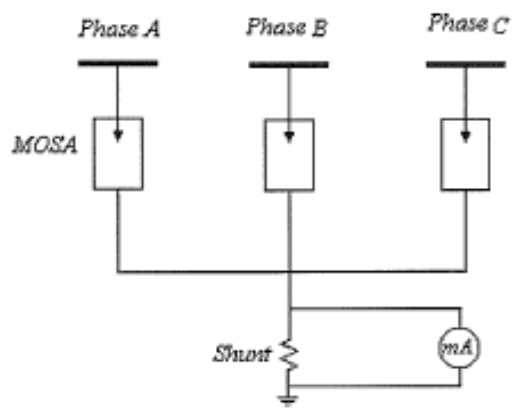

Figure 5 Neutral Current Method

The errors will take place due to unbalancing threephase voltage. Unbalance of the three phase voltages will result in quite misleading results, even with identical MOSA in three phases. In this case, the sum of positive and negative components will not be zero. The non-zero sum will add to the zero sequence components, and an unacceptable neutral current will be displayed by the $\mathrm{mA}$ meter. Due to the non-linear characteristics of MOSA valve elements, not only will the fundamental component of the applied voltage result in the $3^{\text {rd }}$ harmonic resistive leakage current, but also the $3^{\text {rd }}, 5^{\text {th }}, 7^{\text {th }}$ etc. Furthermore, if the MOSA in the three phases age identically, this method cannot distinguish whether the increased current is due to MOSA ageing or due to external effects. Thus, in order to make full use of the advantage of this method, modifications need to be introduced and the testing technique needs to be improved.

\subsubsection{Modified Shifted Current Method:}

Modified Shifted Current Method (MSCM) is used to measure or extract the resistive current the applied voltage does not require. The method is totally based on the manipulation of the total leakage current waveform. Total leakage current is summation of resistive leakage current and capacitive leakage current. Where $I_{X}(t)$ the total leakage is current, $I_{X}(t)$ is also a summation of $I_{r}(t)+I_{c}(t)$ which are not in phase with each other and magnitude for both the currents are different. I $x$ shifted is the phase shifted of total leakage current (by a quarter of period of waveform), and $I_{\text {sum }}$ is the summation of the total leakage current and the shifted current wave form. By signal manipulation technique, from this summation current, the capacitive component of total leakage current can be determined, and thereafter the resistive component of the leakage current can also be obtained by subtracting the capacitive component from the total leakage current.

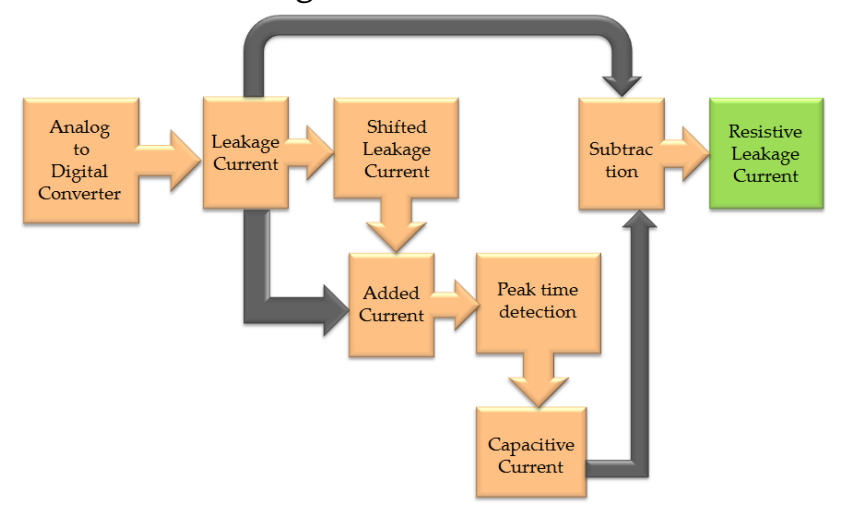

Figure 6 Basic Block Diagram for Modified Shifted Current Measurement 
Block diagram of the algorithm for calculating resistive leakage current using the shifted current method is shown in Figure 6.

Firstly, measure the arrester total leakage current (combination of resistive leakage current + capacitive current) and then shift the measured arrester total LC by quarter period $(1 / 4 t h)$ of its operating frequency. Now in next step both the two total leakage currents are summed together and their peak time determined. The peak time obtained is used to determine the peak time of the capacitive current component. The peak value of the capacitive component is also determined from the original leakage current waveform. By using the determined peak time of total leakage current obtained from summation of measured and shifted leakage current generate the capacitive leakage current. Finally, the resistive leakage current is obtained by subtracting the capacitive leakage current from the measured total leakage current.

Based on the above proposed technique, the algorithm to separate the resistive leakage current from the total leakage current is built. The algorithm for the shifted current method could be summarized as follows.

This method used to extracting the leakage current of the metal oxide surge arrester.

$I_{X}, I_{C}$, and $I_{R}$ Can be written as;

$$
I_{X}(t)=I_{C}(t)+I_{R}(t)
$$

The resistive component can be obtained by;

$$
I_{R}(t)=I_{X}(t)-I_{C}(t)
$$

The subtraction in (13) can be carrying out as below;

$$
I_{X}(t)=I_{x} \cos (\omega t)
$$

where $\left[I_{R}\right]$ is the resistive current component with harmonics.

Shifted the total leakage current by quarter period

$$
I_{x \text { Shifted }}(t)=I_{x} \cos \left[\omega\left(t-\frac{1}{4 f}\right)\right]
$$

$$
I_{\text {sum }}(t)=I_{x}\left[\cos (\omega t)+\cos \left\{\omega\left(t-\frac{1}{4 f}\right)\right\}\right]
$$

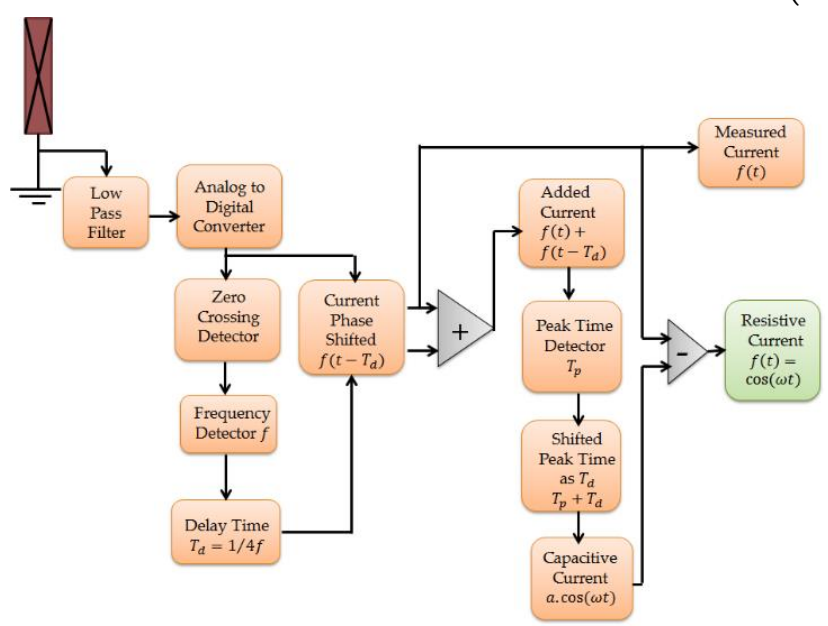

Figure 7 Flow Chart of Modified Shifted Current Method

The algorithm for calculating resistive leakage current using the modified shifted current method is described as follows,

1. Firstly the actual leakage current data is given to the low pass filter and ADC.

2. The digital form of the input data is analyzed and by using phase locked loop the frequency of the signal is determined. A new waveform formed by introducing a quarter period delays to original total leakage current.

3. The total leakage current waveform is added to the delayed waveform.

4. Peak time of the summation waveform is observed. This peak is used to determine the peak time of capacitive current which is a quarter periods delayed from the total leakage current.

5. Based on the information from 3 and 4 , generate a sinusoidal waveform to represent the capacitive leakage current based on peak time, peak value and frequency detected.

6. The resistive leakage current can be obtained by subtracting capacitive leakage current from total leakage current.

Summation equation (14) and (15) 
3.4 Various Instruments Used for Measurement of Resistive Leakage Current from Total Leakage Current

\subsubsection{Leakage current monitor (LCM)}

LCM III is a single channel portable system for condition monitoring of live Metal Oxide Surge Arrestors. The system is suitable for measurement and analysis of Resistive Leakage Current of surge arrestor in live switchyard conditions without any need for shutdown. The system is based on the measurement of $3^{\text {rd }}$ harmonic resistive current with compensation for harmonic in the system voltage as per standard IEC 60099 5. It provides quick and reliable information about the health of the surge arrester and allows for storing of all the measurement data in the field. Customized Reports can be generated and printed on the optional printer. The LCM meter has the provisions to measure total leakage current, third harmonic resistive leakage current, resistive current.

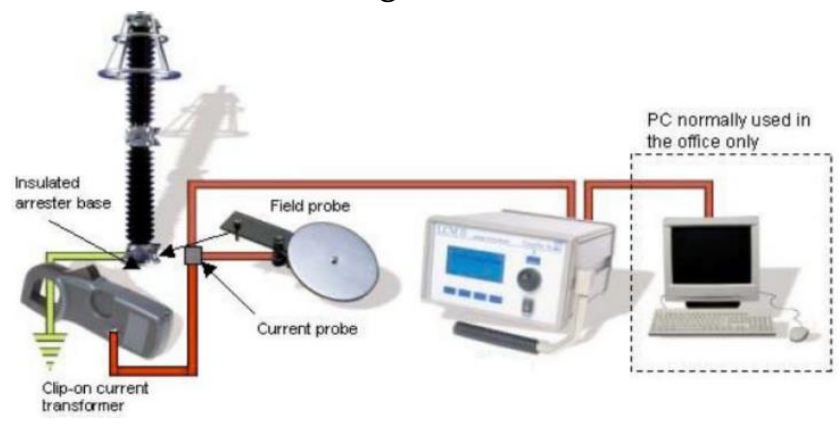

Figure 8 Leakage Current Monitor

For on line monitoring of leakage current, LCM current input terminals are connected across Surge Counter usually installed in Surge Arrestor. For electrical isolation, Clamp on type CT is used to measure current passing through the Surge Arrester without breaking ground return line. Compensation for line voltage 3rd harmonic distortion is derived from HV antenna capacitive pickup voltage divider from Surge Arrestor. Instrument measures total current at fundamental power frequency, resistive component of total current and third harmonic component of leakage current continuously. Instrument can store these measured values in nonvolatile data memory. Frequency of power line is automatically tracked and special narrow band pass filter centered at 3rd harmonic frequency employed for isolation of $3^{\text {rd }}$ harmonic leakage current. Rs232C data Interface is available to transfer recorded values in data memory for transfer to a PC. Since measured current value depends not only on applied voltage on surge arrestor, but also operating temperature, temperature sensor is provided on Instrument rear panel to record temperature also

\section{Discussion and Conclusion}

The electrical properties of surge arrester are discussed. The reasons of increase in resistive leakage current are identified and the diagnoses for these are studied. Various methods are discussed to measure resistive leakage current is discussed. Some of the methods to separate the resistive leakage current were reviewed and all of the methods require voltage measurement in term to obtain the resistive leakage current. The new method based on the cancellation capacitive leakage current component from total leakage current has been done and the method did not require the voltage measurement. The accuracy of the offline method is not good as the accuracy of online method. The Applied voltage measurement is difficult of the system when it is in working condition. So the risk and complexity increases in this type of measurement. There are also problems in online measurement of the current such as in probe based method measurement of probe current. From all the methods discussed above the MSCM method is quite effective and easy to measure the resistive leakage current from total leakage current. The safety issues and complexity problems are eliminated as well the applied voltage is also not required. The Leakage Current Monitor apparatus is also used for measurement of resistive leakage current of surge arrester onsite. There are some risks in measurement but the direct extraction of $3^{\text {rd }}$ harmonic leakage current take place. 


\section{REFERENCES}

[1]. J. Lundquist, L. Stenstorm and A. Schei, B.Hansen 1989. New Method for Measurement of the Resistive Leakage Current of Metal Oxide Surge Arrester in Service. IEEE Power Engineering Society for Presentation.

[2]. Xianglian Yan, Yuanfang Wen, Xiaoyu Yi (2002) . Study on the Resistive Leakage Current Characteristic of MOV Surge Arresters. IEEE conference

[3]. C.A. Spellman et al (1997). A Technique for On-Line Monitoring of $\mathrm{ZnO}$ Surge Arrester. 10th International Symposium on High Voltage Engineering, Canada

[4]. Zulkurnain Abdul-Malek, Novizon Yusoff and Mohd Fairouz Mohd Yousof (2010). Performance Analysis of Modified Shifted Current Method for Surge Arrester Condition Monitoring. IEEE Conference

[5]. Chandana Karawita and M. R. Raghuveer (July 2006). Onsite MOSA Condition Assessment-A New Approach. IEEE Transactions on Power Delivery, Vol. 21, No. 3

[6]. Hanxin Zhu, M.R.Raghuveer (1999). Influence of Harmonics in System Voltage on Metal Oxide Surge Arrester Diagnostics. IEEE Conference on Electrical Insulation and Dielectric Phenomena

[7]. Vegard Larsen and Kjetil Lien (Nov 2007). InService Testing and Diagnosis Of Gapless Metal Oxide Surge Arresters IX International Symposium on Lightning Protection-Brazil

[8]. Handbook on Maintenance instruction for "Condition monitoring of Lightening Arresters provided on traction systems on Indian Railways"

\section{Cite this article as :}

Prof. Samruddhi Shaha, Yaseen Khan Anwar Khan Dakhani, "Various Methods for Measurement of Resistive Leakage Current of Metal Oxide Surge Arrester ", International Journal of Scientific Research in Science, Engineering and Technology (IJSRSET), Online ISSN : 2394-4099, Print ISSN : 2395-1990, Volume 8 Issue 4, pp. 81-89, July-August 2021. Available at doi : https://doi.org/10.32628/IJSRSET218374 Journal URL : https://ijsrset.com/IJSRSET218374 